\title{
NICOLAS DE CUSA: LA PRIORIDAD DEL SIMBOLO MATEMATICO EN LA BUSQUEDA DE LA SABIDURIA
}

Claudia D’Amico*

SÉNTESE - Nicolau de Cusa é conhecido por suas teorias a respeito do conhecimento, principalmente por sua obra $A$ douta ignorância. Nela e em outras, sempre dentro de uma visão medieval, vê-se que a sabedoria cusana é uma tentativa de alcançar incansavelmente o inalcançável. Para tanto são de grande importância os símbolos matemáticos.

PALAVRAS-CHAVE - Nicolau de Cusa, conhecimento, símbolos matemáticos.
ABSTRACT - On account of his theories about the knowledge, specially in the De docta ignorantia, everyone knows Nicholas of Cusa. In this work as in others, always directed by a medieval view, the reader sees that the wisdom of this author attempts to reach indefatigably the inaccessible, giving in this way a great importance to mathematical symbols.

KEY WORDS - Nicholas of Cusa, knowledge, mathematical symbols.

La concepción acerca del saber está signada en el pensamiento de Nicolás de Cusa por la máxima doctrina de la ignorancia. El sentido de la docta ignorantia cusana no es, como se sabe, una visión crítica acerca de los límites del conocimiento humano, sino más bien una posición acerca de la índole propia de la Verdad, una e infinita. Pensador todavía medieval, Nicolás identifica a la Verdad suprema con Dios entendido en sus propios términos como Máximo absoluto. La idea misma de "maximidad", como sabemos, no es comparativa sino superlativa: "máximo", pues, es aquello que no puede ser mayor, razón por la cual es unidad fundante de toda multiplicidad. ${ }^{1}$ Una verdad tal escapa a ser sometida a la operación del conocimiento racional: comparar, establecer proporciones. ${ }^{2}$

* Universidad de Buenos Aires.

1 "Maximum autem boc dico, quo nihil maius esse potest. Abundantia vero uni convenit; coincidit itaque maximitati unitas [...] Maximum itaque absolutum unum est quod est omnia, in quo omnia quia maximum..." De doct.ign. 1, 2.5

2 "Maximum autem hoc dico, quo nihil maius esse potest. Abundantia vero uni convenit; coincidit itaque maximitati unitas [...] Maximum itaque absolutum unum est quod est omnia, in quo omnia quia maximum..." De doct.ign. 1, 2.5

\begin{tabular}{|l|l|l|l|l|l|}
\hline VERITAS & Porto Alegre & v. 43 & $\mathrm{n}^{\mathrm{0}} 3$ & Setembro 1998 & p. 657-663 \\
\hline
\end{tabular}


En el pensamiento cusano, el problema gnoseológico se funda, pues, en el problema metafíco-teológico. La doctrina de la ignorancia es "máxima", precisamente, porque al tratar de lo que no tiene proporción es fundante de todo otro saber.

En efecto, no podemos tratar acerca del saber sin establecer la ignorancia. Sin embargo, no se trata de un punto de partida negativo sino de una nueva posibilidad de acercamiento a la verdad. La Sapientia cusana, que es ignorante, consiste más bien en un "alcanzar inalcanzablemente lo inalcanzable" desde el ámbito de la multiplicidad pero trascendiéndolo en busca de su principio. ${ }^{3}$ Veremos qué papel desempeña en él el conocimiento matemático. Para intentar esclarecerlo no nos internaremos en los desarrollos teóricos de De docta ignorantia y De coniecturis - obras capitales de Nicolás - sino que nos dejaremos conducir por un idiota, ${ }^{4}$ sabio por antonomasia, distante por completo de los "especialistas" del saber, que en busca de la sabiduría nos llevará al mercado, vale decir muy lejos de los muros donde tradicionalmente se custodia este saber. La sabiduría será, pues, la posibilidad de ascender desde el mercado - metáfora de la multiplicidad del mundo - en busca de su verdad o principio infinito y, por tanto, inaccesible. Sin embargo, habrá de mostrarse que tal principio es "figurable" a través del símbolo matemáti$\mathrm{CO}^{5}$

En los diálogos acerca de la sabiduria, un orador, en busca de la sabiduría infinita, observa junto al sabio-ignorante las operaciones que se realizan en el mercado.

El elemento matemático es presentado alli por Nicolás de Cusa como la única posibilidad de transferencia de esas operaciones finitas a un ámbito que no es el infinito buscado pero que, por la índole de sus objetos, guarda cierta "vecindad" con él. Dos nociones aparecen, entonces, en auxilio del idiota: el número que gobierna la búsqueda de la unidad en la alteridad, entendida aquí como búsqueda del principio que rige todas las operaciones que se observan en el mercado; la figura geométrica, símbolo imprescindible para trascender el ámbito de lo finito hacia lo infinito matemático y desde allí prefigurar lo infinito absoluto.

3 "Summa sapientia est haec, ut scias, quomodo in similitudine iam dicta attingitur inattingibile inattingibiliter" De sap. $1,7$.

4 El término latino "idiota" tiene el sentido de ignorante, iletrado y, a veces, laico. Es el personaje principal de una serie de cuatro diálogos agrupados bajo este nombre: De sapientia, 1 y II; De mente y De staticis experimentis, escritos en el año 1450 . En este trabajo abordaremos el papel desempeñado por el símbolo matemático en los dos primeros, es decir en los diálogos acerca de la sabiduria.

5 El clásico estudio de Volkmann-Schluck, Nicolaus Cusanus. Die Philosophie im Übergang vom Mittelalter zur Neuzeit, Frankfurt, 1957, señala la noción de imago tal como está tomada en $D e$ docta ignorantia en referencia al símbolo matemático como aquello que hace posible volver visible mediante una representación una realidad invisible. La imagen (Bild) hace visible la figuratividad (Bildlichkeit) de lo invisible (cfr. especialmente, Parte l, cap. 3: "Die Idee einer symbolischen Re präsentation der Wahrheit durch die Mathematik"). 
Si para el Cusano conocer es establecer proporciones - y, en tal sentido, la praecisio o veritas absoluta, improporcionable, es incognoșcible - la medición de aquello pasible de ser proporcionado otorga un cierto tipo de conocimiento que, por definición, excluye la precisión de la verdad. Conocimiento aproximado de la verdad en la alteridad, medición del espíritu, tal es el sentido de la Mutmassung o coniectura cusana. Este tipo de saber no puede, por su condición, prescindir del número en tanto toda proporción o comparación supone una identidad y una alteridad. ${ }^{6}$ El número se vuelve, pues, un símbolo de la identidad que reside en todas las cosas. ${ }^{7}$

El ignorante nos invita a observar las operaciones que se realizan en el mercado, numerar, medir, pesar, ${ }^{8}$ y a fundar desde esta observación no los principios de una scientia sino a ejercer el ars coniecturalis. ${ }^{9,10}$ Precisamente este ejercio es exclusivo de la ratio y diferencia a los hombre de las bestias. ${ }^{11}$ No obstante, tal arte no consiste en ofrecer un mero catálogo de lo observado sino en encontrar el principio que permanece como fundamento de cada una de estas prácticas. Es evidente que la unidad numérica constituye todo número. Lo mismo sucede con el peso mínimo y la medida mínima respecto del pesar y el medir que son una suerte de unidad que rige su operar. Dos razones al menos hacen de estas unidades el principio de sus respectivas operaciones proporcionales: en primer lugar, la precedencia de la unidad respecto de la multiplicidad; en segundo lugar, la explicitación de esa unidad en toda la serie de la diversidad..$^{12}$

Tomenos el caso de la unidad numérica y la numeración. ${ }^{13}$ El número o unidad es principio de la serie enumerada no sólo en el clásico sentido de arché: en cuanto orígen y gobierno. La unidad es aquello por lo cual (per) en lo cual (in) y a partir de lo cual (ex) todo lo numerable es numerado. No sería posible numerar sin el uno: acaso no es el uno la unidad una sola vez, el dos la unidad dos veces y el

6 "Proportio vero cum convenientiam in aliquo uno simul et alteritatem dicat absque numero intelligi nequit" De doct. ign, 1, 1, 3.

7 "Symbolicum exemplar rerum numerum esse" De coniect., 1, I, 7.

8 "Or.: Video ibi numerari pecunias, in alio angulo ponderari merces, ex opposito mensurari oleum et alia" De sap., l, n.5

9. "Magna vis coniecturalis artis tibi hac via panditur, si hunc denarium explicatorium complicatorie notaveris. Ars enim brevissima est, qua veritas ipsa indagatur" De coniect., I, XI, 60.

10 En un interesantísimo artículo Mme. Quillet ("De l'art des conjectures à la science divine selon Nicolas de Cues" en Miscellanea Mediaevalia, 22/1, 1994, pp.95-106) presenta la distinción medieval entre ars y scientia en referencia ai pensamiento de Nicolás de Cusa. Allí muestra que la noción cusana de "coniectura" va a modificar fundamentalmente esta relación: la scientia verdadera es inalcanzable por lo cual se convierte en no-ciencia o ignorancia; el ars se mueve en el ámbito de la ratio y éste debe ser trascendido. El saber matemático, precisamente, es aquel que opera la unión entre arte y ciencia.

11 "Id.: Haec sunt opera rationis illius, per quam homines bestias antecellunt; nam numerare, ponderare et mensurare bruta nequeunt" De sap., 1, n.5.

12 "Sicut igitur unum est principium numeri, ita est pondus minimum principium ponderandi et mensura minima principium mensurandi. Vocetur igitur pondus illud uncia et mensura petitum. Nonne sicut per unum numeratur, ita per unciam ponderatur et per petitum mensuratur?" ibid. n.6.

13 Lo mismo vale para la medida y el peso mínimo que hacen las veces de unidades cuantitativas minimas en sus ámbitos especificos. 
tres, la unidad tres veces, y así sucesivamente ${ }^{14}$ Ahora bien, aunque nada pueda ser numerado sin el uno, la unidad escapa a la numeración..$^{15}$ La comprensión de la unidad numérica está fuera del ámbito de la proporcionalidad, esto es fuera de la esfera de acción de la ratio.

El número o la unidad numérica nos han conducido hacia un espacio donde ya no es posible la proporción: nos encontramos en el "techo" del conocimiento racional. La matemática ha abierto la mens al ámbito de lo improporcionable, al ámbito de lo infinito, tomando conciencia de su límite infranqueable. La ratio, facultad de la distinción, deja el lugar al intellectus, facultad de la totalidad.

Desde aquí, entonces, ya no se puede escalar por los sucesivos peldaños de la comparación sino que resulta imprescindible dar un salto cualitativo: transfers in altissimma ${ }^{16}$ ordenará el idiota al orador. Sin este traslado o transferencia no se habrá de alcanzar de la única manera posible, i.e. inalcanzablemente, la sabiduría buscada, en sí misma, inalcanzable.

El "transfers in altissima" encierra pues la obligación de trascender este infinito cuantitativo al que nos condujo la matemática para poder escapar de la conjetura. La tarea del sabio es la de comprender incomprensiblemente el Número no numerable, la Medida inconmensurable, el Peso imponderable ${ }^{17}$ que es la unidad oculta de todas las cosas, i.e. aquello por lo cual, en lo cual y a partir de lo cual todas las cosas son. ${ }^{18} \mathrm{El}$ número, en cuanto infinito cuantitativo pues, ha abierto las puertas al infinito simpliciter pues su relación con la serie en la cual se explicita opera como figura de la unidad trascendente explicitada en todo lo real. Más aún, la relación entre el número y la numeración es el símbolo que ha vuelto de alguna manera "visible" la invisible relación entre lo uno y lo múltiple, entre Dios y el mundo o, en técnica terminología cusana, entre lo absoluto y lo contracto. Esta relación por la cual la Unidad contiene o co-implica absolutamente toda la diversidad y, a la vez, se explicita o "explica" en esa diversidad es, como se sabe, la relación complicatio-explicatio. ${ }^{19}$ La presentación de esta doctrina cusana fundamental no es aquí meramente teórica sino el resultado de un ascendente camino conjetural que nos ha conducido desde las actividades del mercado a la prefiguración de la relación entre lo Uno y lo múltiple encontrando en el número la clave para dicha prefiguración.

14 "Nonne unum est unum semel, et duo est unum bis, et tria unum ter, et sic deinceps?" ibidem.

15 "Unitas non attingitur numero, quia numerus est post unum" ibid. n. 6

16 ibid. n. 7

17 Es clara aquí la alusión a Dios como aquel que todo lo hace según Mensura, Numerus, Pondus que aparece en las Escrituras (Sap. 11, 21: "Sed et sine his uno spiritu poterant occidi persecutionem passi ab ipsis factis suis, et dispersi per spiritum virtutis tuae: sed omnia in mensura, et numero, et pondere disposuisti" ) Por otra parte, como se sabe, esta tríada es fundamental en el pensamiento de Agustín y en toda la tradición agustiniana medieval.

18 Nótese el uso del altus en su doble sentido de "alto" y "profundo": este locus "in altissima" no sólo refiere la absoluta trascendencia de Dios sino también su presencia como profunda unidad de todas y cada una de las cosas.

19 No expondremos aquí los fundamentos de esta doctrina cusana, tampoco lo hace Nicolás de Cusa en estos diálogos sobre la sabiduría, sólo nos interesa ver cómo el elemento matemático la simboliza (para su exposición teórica, cfr. especialmente De doct. ign., II, 2 y ss.) 
Los bases que sostienen teóricamente la doctrina de la complicatio-explicatio se encuentran, sin duda, en la intuición central del pensamiento cusano: la coincidentia oppositorum en el Máximo absoluto, infinito y uno. En el corazón de la coincidencia de opuestos se encuentra la clave de la relación entre Dios y el mundo.

En efecto, Nicolás de Cusa establece que el Máximo absoluto es tal porque carece de toda relación y, por esto mismo, nada puede oponérsele. De esta manera, el Máximo absoluto coincide necesariamente con su opuesto, el Mínimo absoluto. ${ }^{20} \mathrm{La}$ coincidencia de opuestos en el Máximo no sólo pone de manifiesto su absoluta unidad y simplicidad, sino que conduce necesariamente a no dejar nada fuera de él, i.e. a caracterizarlo como complicatio absoluta de todas las cosas. ${ }^{21}$ Vemos pues cómo ambas doctrinas son complementarias desde el origen del pensar cusano. Tal complementariedad aparece también en estos diálogos acerca de la sabiduría.

En el camino de búsqueda propuesto por el idiota, la relación complicatioexplicatio se nos ha vuelto "visible" a través del número; esta misma "visión" dió cuenta, a su vez, de la incognoscibilidad de esta relación en términos absolutos. Ahora, otro elemento matemático acudirá en ayuda de nuestro ignorante maestro a la hora de profundizar esta relación y dar cuenta del fundamento que la sostiene, i.e. la coincidentia oppositorum. La posibilidad de "ver" tal coincidencia se encuentra en la figura geométrica.

\section{III}

La transsumptio in infinitum es para el Cusano un método que difiere fundamentalmente de la analogía entre lo finito y lo infinito. No se trata de una progresión desde lo múltiple a lo uno: "finiti ad infinitum nulla proportio". ${ }^{22}$ Como hemos dicho, la sabiduria consiste en aprehender de manera inaprehensible la oculta unidad de lo infinito in similitudine iam dicta, ${ }^{23}$ esto es a partir de la multiplicidad representada por el mercado. Como todo método tiene sus pasos y, de alguna manera, ya los hemos recorrido: de las acciones singulares de numerar, pesar, medir, a la unidad numérica; de la unidad numérica no numerable, al Número innumerado. Del ámbito de la ratio al del intellectus. En el caso de la figura geométrica, precisamente por su condición de figura, al llegar a la aprehensión intelectual habremos dejado atrás dos instancias: la imaginatio y la ratio.

Según el Cusano, así habrá de procederse: Primero deben ser consideradas las figuras finitas con sus propiedades y razones; ${ }^{24}$ en segundo lugar, es necesario trasladar o transferir esa figura a su correspondiente figura infinita;25 por último, ascender aún más alto transladándonos desde cualquier figura infinita hasta el mismo absoluto infinito. ${ }^{26}$

20 cfr. De doct. ign., 1, 2.

21 cfr. ibid., II, 3.

22 De doct. ign. 1, 2.

23 De sap., l, n. 7.

24 "l... primo necesse est figuras mathematicas finitas considerare cum suis passionibus et rationibus" De doct. ign., 1, 12.

25 "I... et ipsas rationes correspondenter ad infinitas tales figuras transferre" ibidem.

26 "Post hec tertio adhuc altius ipsas rationes infinitarum figurarum transsumere ad infinitum simplex absolutissimum etiam ab omni figura" ibidem. 
En este diálogo acerca de la sabiduría, el idiota recrea este procedimiento a propósito de la búsqueda que lo ocupa: la inasible infinitud absoluta. La pregunta, en este caso, se dirige a la rectitudo absoluta ${ }^{27}$ que, como sabemos, es otro nombre para la veritas o la praecisio que es Dios. La figura elegida es el círculo. ${ }^{28} \mathrm{En}$ primer lugar, se considera el círculo finito y se establece una proporción, puesto que nos encontramos en el terreno de la conjetura, entre su curvatura y su diámentro: cuanto mayor sea el círculo, mayor será su diámetro. ${ }^{29}$ Esta relación proporcional puede cuantificarse $\mathrm{y}$, al hacerio se establece un principio relativo que vale para todo círculo: la curvatura es más de tres veces el diámetro. ${ }^{30} \mathrm{Al}$ trasladar esta relación al círculo infinito, es decir a la figura que ya no está sujeta al más y al menos, se advierte que en él su diámetro y su curvatura coinciden en una línea infinita. Advertimos, pues, que la relación proporcional desaparece dando paso a la coincidencia de los términos puestos en relación. A partir de la coincidencia de la curvatura y el diámetro en el círculo infinito podemos inferir que la rectitud conviene a lo infinito. ${ }^{31}$ Resta ahora, entonces, transferir la coincidentia oppositorum, en este caso como rectitud infinita matemática al ámbito de lo infinito simpliciter: dar el salto cualitativo. Así como la línea recta infinita, en cuanto coincidencia de curvatura y diámetro, es principio y medida adecuadísima de todo círculo y también de toda figura figurable - de la misma manera Dios es la Medida no mensurable de todo lo real: es la absoluta coincidentia oppositorum en el cual todas las cosas coinciden como en su verdad.

A su vez, la línea recta infinita contiene en sí todas las figuras y a la vez se encuentra explicitada en todas ellas, en sus diferentes formas: triángulo, cuadrado, círculo $^{32}$ de la misma manera Dios es la complicatio absoluta que contiene todas las cosas no en su singularidad sino como siendo Él mismo y, las cosas son explicatio dei en cuanto su oculta unidad no es otra que éste, su ser en Dios.

Hasta aquí hemos visto las "semejanzas" ("des-semejantes" agregaría el Cusano) que guarda la infinitud cuantitativa de la figura geométrica infinita con la infinitud absoluta: ambas son complicación, precisión, rectitud, verdad, medida y perfección de todo lo por ellas complicado. Sin embargo, no debe perderse de vista la infinita distancia que separa ambos infinitos: la rectitud de la linea infinita implica ya un grado de contracción, un modo de despliegue de la rectitud absoluta como algo determinado. La rectitud absoluta que es Dios es absolutamente ejemplar, precisión, verdad, medida y perfección de todo lo que es. ${ }^{33}$

27 "I... quomodo rectitudini absolutae conveniat infinitas" De sap. II, n. 42.

28 Acerca de la importancia del círculo en el pensamiento cusano cfr. Gaus, J., "Circulus mensurat omnia" en Mensura, Mass, Zahl. Zahlensymbolik im Mittelalter (Misc.Mediaev. 16/2, 1984, p. 435454) especialmente, Parte III.

29 "Tu nosti quod, quanto circulus aliquis fuerit maior, tanto et eius diameter maior" De sap. ㅍ, n.42.

30 ".. .1 cum una sit omnium circulorum curvarum circumferentiarum habitudo diametri ad circumferentiam, quae est habitudo plus quam tripla" ibidem.

31 "Unde infiniti circuli circumferentia foret ex hoc recta, ex quo tibi constat curvitatem, quae recipit magis et minus, non reperiri in infinito, sed solam rectitudinem" ibidem.

32 "Audisti, quomodo linea infinita est circulus. Sic et triangulus, quadrangulus, pentagonus, sic omnes figurae infinitae cum linea infinita coincidunt" ibid., n. 44.

33 "Nam si infinita rectitudo, quae est necessario absoluta, ad lineam contracta reperitur necessario dmnium figurabilium figuram complicatio, praecisio, rectitudo, veritas, mensura et perfectio, tunc absoluta rectitudo absolute penitus et incontracte ad lineam aut aliud quodcumque considerata est similiter absolute monium exemplar, praecisio, veritas, mensura et perfectio" ibid., n. 43. 


\section{IV}

La prioridad del símbolo matemático en el marco de la docta ignorantia cusana despierta cierta perplejidad: ¿cómo conciliar matemática y teología?; ¿es coherente la temática de la tiniebla, del Dios de la teología mística, con este primado? ¿Acaso, hay que entenderlo como fruto de una doble influencia en Nicolás de Cusa: la devotio modema y el descubrimiento en su propio tiempo del poder mensurante de la razón? ${ }^{34}$ Por otra parte si, como fundamentará el Cusano más tarde, los entes matemáticos son una pura creación humana, ${ }^{35}$ ¿es lícito, entonces, realizar una interpretación idealista en cuanto es el hombre quien genera un ámbito propio de cognoscibilidad? ${ }^{36} \mathrm{Si}$ se pretende sostener esto ¿qué relación tendría este ámbito con el de la verdad, toda vez que las cosas, según Nicolás de Cuśa, están sólo en la mente divina como en su precisión o verdad? ${ }^{37}$

El número y la figura geométrica substraen a la cosa su mutabilidad y ofrecen una cierta praecisio. La matemática constituye, pues, la más perfecta de las artes, el límite superior posible en el ámbito de la ratio donde la verdad y la precisión son siempre conjeturales. La praecisio matemática es sólo, entonces, imagen de la praecisio de la ciencia divina inaccesible donde todas las cosas son en su verdad. Nuestra ignorancia, entonces, no concierne sólo a la quididad divina sino a la quididad de todas y cada una de las cosas. Y asi como si pudiérmos conocer a Dios, por esto mismo conoceríamos todas las cosas; de la misma manera, si pudiéramos conocer con absoluta precisión sólo una de las cosas que se cuentan, miden o pesan en el mercado, conoceríamos a Dios.

Por ahora sólo no es dado intuir el misterio de la eterna sabiduría a través de un medio enigmático "sin el cual en este mundo es imposible tener una visión de Dios". Este enigma no es sino el mundo mismo, libro que Dios ha escrito con su propia mano. El ámbito de la diversidad, pues, no es construído por el hombre como el ámbito de lo que es posible conocer, sino que es un enigma que encierra algo uno, infinito y absoluto. Ese infinito que trasciende la diversidad y, a la vez constiuye el entramado del mundo, es parcialmente. decodificable en caracteres matemáticos. Su autor, trascendente-inmanente, permanece oculto "hasta que É1 nos conceda que sin enigma se nos vuelva visible". ${ }^{38}$

34 Preguntas que, de alguna manera, se desprenden del trabajo de J. Quillet, op. cit., p. 105-6. cfr. De mente, III: "Id: Tu nosti, orator, quomodo nos exserimus ex vi mentis mathematicales figuras" (f. $83 \mathrm{v}$ ).

36 A la manera en que lo hace la ya clásica interpretación de Cassirer.

37 cfr. al respecto la crítica que realiza a la interpretación de Cassirer, Muraro Vaiani, L., "Congettura e precisione matematica in Nicolò Cusano" en Riv.di filosofia neo-scolastica, LXII / 1-2, 1970, p. 163-172.

"Sic nunc habes id, quod in aetema sapientia contemplari conceditur, ut intuearis omnia in simplicissima rectitudine verissime, praecisissime, inconfuse et perfectissime, licet medio aenigmatico, sine quo in hoc mundo dei visio esse nequit, quousque concesserit deus, ut absque aenigmate nobis visibilis reddatur" De sap. I, n. 47. 